\title{
Tandem-repeat protein domains across the tree of life
}

Kristin Jernigan, Seth Bordenstein

Tandem-repeat protein domains, composed of repeated units of conserved stretches of 2040 amino acids, are required for a wide array of biological functions. Despite their diverse and fundamental functions, there has been no comprehensive assessment of their taxonomic distribution, incidence, and associations with organismal lifestyle and phylogeny. In this study, we assess for the first time the abundance of armadillo (ARM) and tetratricopeptide (TPR) repeat domains across all three domains in the tree of life and compare the results to our previous analysis on ankyrin (ANK) repeat domains in this journal. All eukaryotes and a majority of the bacterial and archaeal genomes analyzed have a minimum of one TPR and ARM repeat. In eukaryotes, the fraction of ARM-containing proteins is approximately double that of TPR and ANK-containing proteins, whereas bacteria and archaea are enriched in TPR-containing proteins relative to ARM- and ANKcontaining proteins. We show in bacteria that phylogenetic history, rather than lifestyle or pathogenicity, is a predictor of TPR repeat domain abundance, while neither phylogenetic history nor lifestyle predicts ARM repeat domain abundance. Surprisingly, pathogenic bacteria were not enriched in TPR-containing proteins, which have been associated within virulence factors in certain species. Taken together, this comparative analysis provides a newly appreciated view of the prevalence and diversity of multiple types of tandem-repeat protein domains across the tree of life. A central finding of this analysis is that tandem repeat domain-containing proteins are prevalent not just in eukaryotes, but also in bacterial and archaeal species. 
1 Kristin. K. Jernigan

2 Department of Cell and Developmental Biology, Vanderbilt University, Nashville, Tennessee

3 37232, United States of America

4 Seth R. Bordenstein

5 Department of Biological Sciences, Vanderbilt University, Nashville, Tennessee 37232, United

6 States of America

7 Department of Pathology, Microbiology, and Immunology, Vanderbilt University, Nashville,

8 Tennessee 37232, United States of America

9 Corresponding author:

10 Seth R. Bordenstein

11 Address: U7215 BSB / MRB III, 465 21st Ave South, Nashville, TN 37232

12 Phone: (615) 322-9087

13 Email: s.bordenstein@vanderbilt.edu 


\section{Introduction}

While many functional protein domains exist, tandem-repeat domains are one of the most

16 abundant classes of protein-protein interaction domains (Heringa 1998; Marcotte et al. 1999).

17 Tandem-repeat domains are comprised of 'tandem' arrays of repeating units of approximately 20-

1840 amino acids that contain simple structural motifs, such as $\alpha$-helices or $\beta$-sheets (Kobe \&

19 Kajava 2000). These domains can be classified as having either an open structure with a variable

20 number of repeats or a closed structure with a fixed number of repeats. The repeat domains that

21 fall in the former category are typically composed of 3-20 or more repeats, and the resulting

22 structural formation determined by the tandem array of these protein motifs provides a platform

23 for protein-protein interactions (Grove, Cortajarena, Regan 2008). Some of the most common

24 open-structure repeat domains include the ankyrin (ANK), tetratricopeptide (TPR), and armadillo

25 (ARM) domains.

26 Tandem-repeat containing proteins are present in all domains of life and function in

27 nearly every cellular process from transcriptional regulation in the nucleus to cell adhesion at the 28 plasma membrane (Andrade et al. 2001). Based our recent study on the distribution of ANK-

29 containing proteins across the tree of Life, ANK domains are more common in bacteria (51\% of

30 strains) and archaea (11\% of strains) than previously recognized (Jernigan \& Bordenstein 2014).

31 Here, we set out to determine the distribution of TPR and ARM repeats across the three domains

32 of life to examine three general patterns: (i) the origins and distribution of these repeats across the

33 tree of life, (ii) the correlated presence of these repeats in each proteome, and (iii) the strength of

34 associations between the presence of these protein domains and the taxonomic lifestyle or

35 phylogenetic relationships of the organisms that they inhabit. We establish that these tandem-

36 repeat proteins are not only present, but abundant in all domains of the universal tree of life. 
The ANK repeat is a 33 amino acid motif that originally was discovered in

38 Saccharomyces cerevisiae, Schizosaccharomyces pombe, and Drosophila melanogaster and was

39 named after a human protein by the same name (Breeden \& Nasmyth 1987; Mosavi et al. 2004).

40 The structure of a single motif begins with a $\beta$-turn that precedes two antiparallel $\alpha$-helices and

41 ends with a loop that feeds into the next repeat (Fig. 1A). These motifs stack one upon another to

42 form an ANK domain and provide an interface for interacting with other proteins (Gorina \&

43 Pavletich 1996; Sedgwick \& Smerdon 1999). ANK-containing proteins have been identified in

44 all domains of life, and special attention has recently been paid to the role of ANK-containing

45 proteins in host-microbe interactions in bacterial species such as Legionella pneumophila (Al-

46 Khodor et al. 2010; de Felipe et al. 2008), Anaplasma phagocytophilum (JW et al. 2007), and

47 Ehrlichia chaffeenis (Zhu et al. 2009).

The TPR repeat is 34 amino acids long and is composed of two $\alpha$-helices producing an $\alpha$ helix-turn- $\alpha$-helix motif (Fig. 1A) (Das et al. 1998). First identified in yeast cell cycle proteins, it 50 was coined the tetratricopeptide repeat for its 34 amino acid sequence (Hirano et al. 1990;

51 Sikorski et al. 1990). A typical TPR domain contains between 3-16 TPR repeats and ends with

52 one additional resolving $\alpha$-helix that is thought to provide stability to the protein domain

53 (D'Andrea \& Regan 2003). TPR-containing proteins occur in all domains of life (Cerveny et al.

54 2013; Ponting et al. 1999), but no systematic investigation has specified their general distribution

55 and incidence across the universal tree.

56 ARM repeats, at 42 amino acids, are composed of three $\alpha$-helices (Fig. 1A) (Tewari et al.

57 2010). This domain was first identified in the Drosophila melanogaster segment polarity protein,

58 Armadillo (Peifer et al. 1994). In our analysis, other repeat domains with similar sequence, 
59 structure, and function are classified under the ARM repeat superfamily, including the HEAT

60 repeat (Andrade et al. 2001; SUPERFAMILY). The HEAT repeat, named after the first proteins

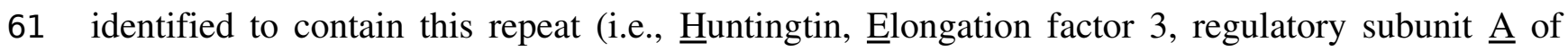

62 Protein Phosphatase 2A, and Target of rapamycin) is composed of two $\alpha$-helices (Andrade \&

63 Bork 1995; Andrade et al. 2001). Although composed of a different number of $\alpha$-helices, both

64 repeats produce a similar concave surface important for protein interactions. Sequence analysis

65 indicates that both repeats contain seven conserved amino acids (Andrade et al. 2001; Cingolani

66 et al. 1999; Eklof Spink et al. 2001; Lee et al. 2003).

67 All three repeat domains are composed of multiple repeated units of relatively simple 68 protein motifs that impart important cellular functions. To the best of our knowledge, this is the 69 first comprehensive analysis of multiple repeat domains across the tree of life that additionally 70 shows how their abundance associates with phylogenetic history and lifestyle

\section{Materials and Methods}

72 ANK-containing Protein Data Acquisition and Analysis

73 All genome information was obtained from the SUPERFAMILY v1.75 database

74 (SUPERFAMILY ; Wilson et al. 2009), including the taxonomy, and number of ANK, TPR and

75 ARM-containing proteins. At the time of the analysis, the SUPERFAMILY database contained

76 protein domain information on 2,489 strains, where there can be more than one strain 77 representing a single phylogenetic species. This database is an archive of structural and 
78 functional domains in proteins of sequenced genomes (Wilson et al. 2009), which are annotated

79 using hidden Markov models through the SCOP (Structural Classification of Proteins)

80 SUPERFAMILY protein domain classification (Gough et al. 2001; SUPERFAMILY). We note

81 appropriate caution that ANK, TPR, and ARM domains are called based on a computational

82 framework and are not experimentally confirmed. NCBI's Genome Resource was used to obtain

83 total gene and protein numbers for each organism in the analysis (Table S1). To determine the

84 percent of an organism's total protein number (proteome) that is composed of ANK/TPR/ARM-

85 containing proteins, the number of ANK/TPR/ARM-containing proteins was divided by the total

86 number of proteins and multiplied by 100 . Only organisms with available total protein

87 information were used in this analysis. For these analyses, an average of the number and/or

88 percent of ANK/TPR/ARM-containing proteins for all strains of the same species were used.

89 16S rRNA Phylogenetic Tree and Independence Analysis

90 16S rRNA sequences from one randomly selected species in each class of Fig. 3 were aligned by

91 MUSCLE in Geneious Pro 5.6.2. Prior to building the tree, a DNA substitution model for the

92 alignment was selected using jModelTest version 2.1.3 (Darriba et al. 2012; Guindon \& Gascuel

93 2003). A Bayesian phylogenetic tree was generated by MrBayes using the HKY85 model of

94 DNA sequence evolution (Hasegawa et al. 1985; Huelsenbeck \& Ronquist 2001; Ronquist \&

95 Huelsenbeck 2003). For testing phylogenetic independence of ANK/ARM/TRP-containing

96 proteins in bacteria, the PDAP program in Mesquite was used to generate independent contrasts

97 for the data in Fig. 5 (Maddison \& Maddison 2006; Midford et al. 2005). Phylogenetic 
98 Independence version 2.0 (Reeve \& Abouheif 2003) performed the Test For Serial Independence 99 (TFSI) based on the Bayesian tree.

100 Results

101 The Incidence of Tandem-Repeat Containing Proteins Decreases From Eukaryotes > Bacteria > 102 Archaea

Of the 2,489 proteomes analyzed, 1,911 are from the domain Bacteria, 445 are from the

104 domain Eukarya, and 133 are from the domain Archaea. For many species of bacteria and 105 archaea, more than one proteome per species is present within the SUPERFAMILY database. We 106 report that all eukaryotic species contain at least one TPR, ANK, and ARM-containing protein 107 (except Saccharomyces cerevisiae CLIB382, which lacks an ANK-containing protein and a 108 completely annotated genome) (Fig. 1B, Table S1). Nearly all species of bacteria (96.42\%, 109 970/1007) and a majority of the archaeal species $(80.73 \%, 88 / 109)$ contain at least one TPR110 containing protein. Similarly, we found that a majority of bacterial and archaeal species $(77.73 \%$ 111 and $80.73 \%$, or 782/1007 and 88/109, respectively) contain at least one 1 ARM-containing 112 protein. ANK-containing proteins were the least abundant protein domain with 56.6\% (569/1007) 113 of bacterial species and only $13.76 \%$ (15/1007) of archaeal species containing one or more ANK114 containing proteins (Fig. 1B).

115 When we group organisms into genera to account for biases in species selected for 116 sequencing, we find no major changes in the distribution and incidence of these proteins. $98.2 \%$ 117 of bacterial genera (531/541) and $77.9 \%$ of archaeal genera (53/68) contain species that encode at 118 least one TPR-containing protein. $76.8 \%$ of bacterial genera (417/541) and $77.1 \%$ of archaeal 
119 genera (54/68) contain species that encode at least one ARM-containing protein. Finally, 56.7\%

120 of bacterial genera (307/541) and $8.8 \%$ of archaeal genera (6/68) contain species that encode at

121 least one ANK-containing protein.

122 Next, we normalized the percent of the proteome dedicated to each protein type across the 123 three cellular domains to compare differences in protein abundance (Table S1, Fig. 2). After 124 normalizing for proteome size, we find that eukaryotes consistently have higher amounts of each 125 protein domain than the other two domains, but the relative fraction of each protein in the specific 126 domains varies. For instance, the fraction of ARM-containing proteins is approximately double 127 the abundance of TPR and ANK-containing proteins in eukaryotes (Fig. 2A, Mann-Whitney U, $p$ $128<0.0001$, ANOVA, $p=5.79 \mathrm{E}-40$ ); but the enrichment patterns switch in bacteria and archaea, in 129 which the fraction of TPR-containing proteins are enriched relative to ARM and ANK-containing 130 proteins (Bacteria: Mann Whitney U-test $p<0.0001$ for all comparisons, ANOVA $p=2.3 \mathrm{E}-203$. 131 Archaea: Mann Whitney U-test, $p<0.005$ for all comparisons, ANOVA $p=9.85 \mathrm{E}-07$ ).

132 The Variation in Intraproteomic Abundance of Each Repeat Domain is Correlated in Bacteria

133 The majority of bacterial taxa have ANK, TPR, and ARM domains (Fig. 1), which 134 permits an analysis of whether their relative and absolute abundances within each proteome are 135 correlated positively or negatively. We performed non-parametric correlation analyses on the 136 normalized percent and absolute number of each protein per species. Results specify significant 137 correlations for (i) the intraproteomic relative abundance of each domain-contain protein across 138 numerous bacterial species (Fig. 3A) and (ii) the intraproteomic absolute abundance (i.e., total 139 number of domain-containing proteins) (Fig. 3B). The stronger correlations, as measured by the 
140 Rho values, occur in analyses when the TPR domain is compared to the ANK and ARM

141 domains.

142 To determine which bacterial taxa are enriched for ANK, TPR, and ARM-containing

143 proteins, we analyzed 24 bacterial classes composed of 953 proteomes for the percent of species

144 in each class that have one or more domain containing proteins (Fig. 4). As noted above, most

145 bacterial species have at least one TPR domain, and all of the classes analyzed contain species

146 with one ARM domain (Fig. 4). While the percent of species in a bacterial class that have one or

147 more domains can vary widely, the Mollicutes curiously show low abundance for each of the

148 domains (range of 3.1\%-12.5\%, Fig. 4). We discuss this outlying taxa below.

The bacteria that harbor an enriched fraction of ANK, TPR, and ARM-containing

150 proteins, as defined by the species that fall within the top $15 \%$ of protein domain abundance, are

151 shown adjacent to the bacterial tree in Fig. 5. For the ANK and ARM-containing proteins, the

152 percent of the species that meet this enrichment cutoff are not significantly associated with the

153 bacterial tree such that the normalized abundance of ANK and ARM-containing proteins per

154 proteome across the 24 classes is independent of phylogenetic relatedness (ANK $p=0.5590$,

155 ARM $p=0.3770$, PI test, (Reeve \& Abouheif 2003)) (Fig. 5). In contrast, normalized TPR-

156 containing protein abundance is phylogenetically dependent $(p=0.0270$, PI test). This

157 association appears to be due to the abundance of TPR-containing proteins in the classes

158 Bacteroidia, Flavobacteria, Cytophagia, and Sphingobacteria, which are all part of the phylum

159 Bacteroidetes. To test if the phylogenetic dependence of the TPR abundance is robust to small 160 changes in the cutoff value, we repeated the analysis with the top $10 \%$ and $20 \%$ of bacterial taxa

161 that harbor an enriched fraction of TPR-containing proteins. We find phylogenetic dependence at 
162 the $10 \%$ cutoff ( $p=0.002$, PI test), but not at the $20 \%$ cutoff ( $p=0.0780$, PI test) (data not 163 shown).

Finally, the percent of species in each class with an abundance of ANK and ARM-

165 containing proteins is independent of phylogenetic history (Fig. 5). These values are correlated

166 to the percent of species with a high abundance of TPR-containing proteins, but are not correlated 167 to each other (Fig. 5C).

We reported in an article published previously in this journal that host-associated bacteria

170 (including obligate intracellular bacteria that replicate strictly within host cells and facultative

171 host-associated bacteria) are enriched in ANK-containing proteins in comparison to free living

172 bacterial species that solely replicate outside of host cells (Jernigan \& Bordenstein 2014). To test

173 if host-associated bacterial species are preferentially enriched in ARM-containing proteins, we

174 examined the abundance of ARM-containing proteins per strain. The percent of bacterial species

175 with a high ARM-containing protein composition rapidly declines as the percent of ARM-

176 containing proteins increases (Fig. S1). Thus, we assigned one of three lifestyle annotations (free-

177 living (FL), facultative host-associated (FHA) and obligate intracellular (O) bacteria) for all

178 bacterial species with at least $0.2 \%$ of their proteome dedicated to ARM-containing proteins

179 (representing the top 7.5\% of species enriched in ARM-containing proteins) (Table S2). We

180 selected this cut off for annotation of the lifestyles to analyze bacterial species whose proteome is

181 enriched in ARM-containing proteins. When analyzing absolute protein abundance, we found

182 that host-associated microbes (i.e., obligate intracellular and facultative host-associated) contain, 
183 on average, a lower number of ARM-containing proteins than those that are free-living (Fig. 6A).

184 However, once normalized for genome size, the difference is not statistically significant (Fig. $1856 \mathrm{~B})$.

Similarly, to determine if host-associated bacteria are enriched in TPR-containing

187 proteins, we assigned these three lifestyle annotations for all bacterial species with at least $1.5 \%$

188 of their proteome dedicated to TPR-containing proteins (representing the top $8.2 \%$ of species

189 enriched in TPR-containing proteins) (Table S3). The percent of bacterial species also declines as

190 the cutoff percentage of TPR-containing proteins nears 1.5\% (Fig. S2), so we analyzed the

191 lifestyle of species within this cutoff. Once again, the proteomes of host-associated bacteria are 192 not enriched in TPR-containing proteins (Fig. S3).

\section{TPR Domains Are Not Enriched in Pathogenic Bacteria}

194 Roles for bacterial TPR-containing proteins in virulence mechanisms have gained 195 increasing attention over the past decade (Cerveny et al. 2013), spanning adhesion of Francisella 196 tularensis to host cells through pili formation (Chakraborty et al. 2008), translocation of 197 Pseudomonas aeruginosa effectors into host cells (Broms et al. 2006), and inhibition of 198 phagolysosomal degradation of Mycobacterium tuberculosis (Chao et al. 2010). Thus, we 199 hypothesized that the relative abundance of TPR-containing proteins in bacterial proteomes 200 would be associated with increased virulence. To determine if pathogenic bacteria were enriched 201 in TPR-containing proteins, we generated a list of bacterial species with TPR-containing protein 202 enriched proteomes (i.e., species with a TPR proteome composition greater than $2.0 \%, \mathrm{~N}=48$ 203 species, in comparison to the bacteria-wide average of $0.66 \%$ ). Based on primary literature 
204 searches, we categorized these taxa by their lifestyle and whether or not they cause human 205 disease. Interestingly, only $22.9 \%$ of these taxa enriched in TPR-containing proteins were 206 pathogenic (Table S4), and those species that are pathogenic have not been documented to harbor 207 virulence factors that contain TPR domains.

208 We also analyzed the number of TPR-containing proteins present in bacterial species 209 known to harbor TPR containing-virulence factors as described by Cerveny et. al (Cerveny et al. 210 2013) (Table S5). We report that these species have a relatively reduced number and proteome 211 composition of TPR-containing proteins (Table S5). Many of these species contained fewer 212 TPR-containing proteins than the average of all species in the data set (average $13.2 \pm 8.16 \mathrm{SD}$ 213 vs. $23.8 \pm 28.23$ SD for the whole data set, T-test, $\mathrm{p}=0.0008)$.

\section{Discussion}

217 bacterial and archaeal taxa analyzed (96.42\% and $80.73 \%$, respectively) contain at least one TPR218 containing protein. In bacterial species, $85.6 \%$ of all species analyzed contain at least two repeat 219 domains, and of those species, over half (52\%) contain all three repeat domains. Interestingly,

220 Mollicutes are the only bacterial class with a reduced protein composition for all three domain221 containing proteins. Of the 32 Mollicutes species, one of these species contains an ARM222 containing protein, one contains an ANK- containing protein, and four contain a TPR-containing 223 domain. This observation may, in part, be due to the reduced genome sizes and simplified 
224 metabolic pathways of Mollicutes, though many obligate intracellular bacteria with similar traits

225 have these protein domains. Mollicutes are relatively unique among bacteria in that they also lack

226 peptidoglycan, and instead have cholesterol-containing cell membranes (Krieg et al. 2011).

227 Although it is uncertain if the highly reduced abundance of tandem repeat domain-containing

228 proteins in this group is linked to the absence of peptidoglycan, there is evidence for an

229 association in Escherichia coli in which the TPR-containing lipoprotein LpoA stimulates the

230 major peptidoglycan synthase PBP1A (Jean et al. 2014).

231 We express appropriate caution that since certain strains of bacteria with relevance to

232 human health have received extra attention in sampling, it is possible that the SUPERFAMILY

233 dataset is not representative of the microbial diversity of the natural world, but rather is enriched

234 in bacterial species that affect human health.

235 TPR-containing Proteins in Bacteria

236 Our analysis indicates that although TPR domains are present in bacterial virulence

237 factors, the abundance of TPR-containing proteins in a proteome is not indicative of virulence.

238 Rather, the specific function of an individual TPR-containing protein likely determines its

239 capacity for virulence. This conclusion is consistent with the variable nature of the amino acid

240 sequence of the TPR repeats, which ultimately determines the interaction profile of the protein

241 (D'Andrea \& Regan 2003). Further structural and molecular analysis of these domains within

242 bacterial virulence factors has been suggested as a means to identify novel antibacterial drugs or

243 the design of attenuated live vaccines that target the interaction between the host's cellular

244 machinery and bacterial TPR domain (Cerveny et al. 2013). 


\section{ARM-containing Proteins in Bacteria}

In our analysis, the ARM superfamily is composed of 23 families of repeat domains (SUPERFAMILY). Many of these domains are composed of conserved structural features such that specific sequence and structural analysis is required to determine the exact identity of the repeat (Andrade et al. 2001). Within bacterial species, a HEAT-like domain, specifically the PBS lyase HEAT-like repeat, was the most common of the 23 families of ARM domains present in the ARM superfamily (Fig. S4). This domain is known to be present in phycocyanobilin lyases, which are a major component of phycobilisomes, a light harvesting complex in cyanobacteria (Fairchild \& Glazer 1994). However, this domain is also present in representative strains of all 25 bacterial classes analyzed (Table S6). PBS lyase HEAT-like domains are also present in many archaeal strains (7/8 analyzed, Table S7), and these domain-containing proteins function in chemotaxis as well as modify tRNA (Miles et al. 2011; Schlesner et al. 2009). It is important to note that the identification of ARM domains by prediction algorithms has been difficult and often misleading due to sequence variability within repeats, and that individual repeat analysis is important (Kippert \& Gerloff 2004; Kippert \& Gerloff 2009).

\section{Repeat Domains in Archaea}

Similar to bacteria, a majority of archaea taxa contained tandem-repeat proteins and have, on average, a greater abundance of TPR-containing proteins than ARM and ANK-containing proteins (Figs. 1 and 2). However, archaea have a greater abundance of ARM-containing proteins, on average, than do bacterial species (Fig. 2B and 2C). The only class that includes 
265 species with no ARM or TPR-containing proteins is the Thermoprotei. While we are unsure of

266 the proximate cause, this anomaly could be due to a phylogenetic contingency as Thermoprotei is 267 the single class in our data set that is part of the phylum Crenarchaeota, rather than 268 Euryarchaeota.

\section{Evolution of Tandem Repeats}

270 The precise number of repeats in a repeat domain, and their amino acid sequences are

271 often variable between orthologs, although specific residues critical for the structure of the motifs

272 remain conserved (Javadi \& Itzhaki 2013). The variation in repeat number is presumed to occur

273 through tandem repeat domain duplication via recombination events resulting in repeat expansion

274 (Andrade et al. 2001; Marcotte et al. 1999). A study on the evolution of the ANK-containing

275 genes in Wolbachia endosymbionts of Drosophila indicated that both homologous and

276 illegitimate recombination, as well as genomic flux (i.e., bacteriophage and transposable

277 elements) were responsible for generating sequence variability between ANK-containing gene

278 orthologs (Siozios et al. 2013). This variability has been utilized as a diagnostic tool for

279 Wolbachia strain fingerprinting (Riegler et al. 2012). Some repeat domain families, including the

280 ANK domain, have been found to duplicate consistent numbers of domains instead of duplicating

281 one repeat at a time (Björklund et al. 2006). Since repeat domains are defined by their collective

282 structure rather than the exact amino acid sequence of each repeat, a substantial amount of

283 variation, or degeneracy, within a single repeat after duplication can be maintained without

284 threatening the integrity of the protein domain (Andrade et al. 2001). 
286 than non-repeat containing proteins, which may provide the organism with a relatively quick 287 means of adapting to new environments (Marcotte et al. 1999). Eukaryotes that contain more 288 repeat-domain containing proteins than either bacteria or archaea may benefit from the increased 289 sequence diversity (Marcotte et al. 1999). For example, the rapid evolution of leucine-rich 290 repeats (LRR), a repeat domain present in many plant resistance (R) genes, appears to drive 291 adaptations in plant innate immune systems (Ellis et al. 2000; Liu et al. 2007). In contrast to this 292 general theory, a recent analysis on the evolution of tandem-repeat proteins in humans found that 293 none of the analyzed proteins underwent a recent duplication or deletion, and a majority of 294 human tandem repeat proteins (61\%) were strongly conserved among all mammals (Schaper et 295 al. 2014). Additionally, the substitution rate of tandem repeats themselves was 2.3 times lower 296 than the sequence surrounding the repeat indicating that the exact sequence of a protein's tandem 297 repeat, and its subsequent structure and function, was conserved over time (Schaper et al. 2014). 298 Either taxa vary in their adaptive capacity via repeat domain changes, or changes in individual 299 repeat domains of specific proteins are advantageous during bouts of adaptation. Whether or not repeat domains in bacteria and archaea evolved through convergent 301 evolution, by descent with modification from a common ancestor, or acquired through horizontal 302 gene transfer (HGT) has yet to be resolved. An analysis of the functional classes of eukaryotic, 303 bacterial and archaeal repeat containing proteins showed that the more ancient the protein class 304 shared between the domains of life, the more likely it would lack repeat proteins, and protein 305 classes that were unique to eukaryotes were the most likely to contain repeat domains (Marcotte 306 et al. 1999). This observation suggests that the acquisition of these repeat domains was a 307 relatively recent evolutionary event. However, due to the apparent abundance of TPR repeats in 
308 all domains of life, it is likely that TPR domains did not independently evolve through

309 convergent evolution (Andrade et al. 2001; Ponting et al. 1999). It was also originally postulated

310 that bacterial ANK-containing proteins were obtained through HGT events, although this does

311 not appear to explain how archaea and non-host associated microbes obtained their ANK-

312 containing proteins (Al-Khodor et al. 2010; Bork 1993; Jernigan \& Bordenstein 2014). Taken

313 together with our analysis here, it does not appear that there is a specific mechanism for the

314 evolution of all repeat domains in bacteria and archaea.

\section{References}

316 Al-Khodor S, Price CT, Kalia A, and Abu Kwaik Y. 2010. Functional diversity of ankyrin repeats in microbial proteins. Trends Microbiol 18:132-139.

Andrade MA, and Bork P. 1995. HEAT repeats in the Huntington's disease protein. Nat Genet 11:115-116.

Andrade MA, Petosa C, O'Donoghue SI, Müller CW, and Bork P. 2001. Comparison of ARM and HEAT protein repeats. J Mol Biol 309:1-18.

Björklund AK, Ekman D, and Elofsson A. 2006. Expansion of protein domain repeats. PLoS Comput Biol 2:e114.

Bork P. 1993. Hundreds of ankyrin-like repeats in functionally diverse proteins: mobile modules that cross phyla horizontally? Proteins 17:363-374.

Breeden L, and Nasmyth K. 1987. Similarity between cell-cycle genes of budding yeast and fission yeast and the Notch gene of Drosophila. Nature 329:651-654.

Broms JE, Edqvist PJ, Forsberg A, and Francis MS. 2006. Tetratricopeptide repeats are essential for PcrH chaperone function in Pseudomonas aeruginosa type III secretion. FEMS Microbiol Lett 256:57-66.

Cerveny L, Straskova A, Dankova V, Hartlova A, Ceckova M, Staud F, and Stulik J. 2013. Tetratricopeptide repeat motifs in the world of bacterial pathogens: role in virulence mechanisms. Infect Immun 81:629-635.

Chakraborty S, Monfett M, Maier TM, Benach JL, Frank DW, and Thanassi DG. 2008. Type IV pili in Francisella tularensis: roles of pilF and pilT in fiber assembly, host cell adherence, and virulence. Infect Immun 76:2852-2861.

Chao J, Wong D, Zheng X, Poirier V, Bach H, Hmama Z, and Av-Gay Y. 2010. Protein kinase and phosphatase signaling in Mycobacterium tuberculosis physiology and pathogenesis. Biochim Biophys Acta 1804:620-627. 
340

341

342

343

344

345

346

347

348

349

350

351

352

353

354

355

356

357

358

359

360

361

362

363

364

365

366

367

368

369

370

371

372

373

374

375

376

377

378

379

380

381

382

383

384

Cingolani G, Petosa C, Weis K, and Müller CW. 1999. Structure of importin-beta bound to the IBB domain of importin-alpha. Nature 399:221-229.

D'Andrea LD, and Regan L. 2003. TPR proteins: the versatile helix. Trends Biochem Sci 28:655662.

Darriba D, Taboada GL, Doallo R, and Posada D. 2012. jModelTest 2: more models, new heuristics and parallel computing. Nat Methods 9:772.

Das AK, Cohen PW, and Barford D. 1998. The structure of the tetratricopeptide repeats of protein phosphatase 5: implications for TPR-mediated protein-protein interactions. EMBO J 17:1192-1199.

de Felipe KS, Glover RT, Charpentier X, Anderson OR, Reyes M, Pericone CD, and Shuman HA. 2008. Legionella eukaryotic-like type IV substrates interfere with organelle trafficking. PLoS Pathog 4:e1000117.

Eklof Spink K, Fridman SG, and Weis WI. 2001. Molecular mechanisms of beta-catenin recognition by adenomatous polyposis coli revealed by the structure of an APC-betacatenin complex. EMBO J 20:6203-6212.

Ellis J, Dodds P, and Pryor T. 2000. Structure, function and evolution of plant disease resistance genes. Curr Opin Plant Biol 3:278-284.

Fairchild CD, and Glazer AN. 1994. Oligomeric structure, enzyme kinetics, and substrate specificity of the phycocyanin alpha subunit phycocyanobilin lyase. J Biol Chem 269:8686-8694.

Gorina S, and Pavletich NP. 1996. Structure of the p53 tumor suppressor bound to the ankyrin and SH3 domains of 53BP2. Science 274:1001-1005.

Gough J, Karplus K, Hughey R, and Chothia C. 2001. Assignment of homology to genome sequences using a library of hidden Markov models that represent all proteins of known structure. J Mol Biol 313:903-919.

Guindon S, and Gascuel O. 2003. A simple, fast, and accurate algorithm to estimate large phylogenies by maximum likelihood. Syst Biol 52:696-704.

Hasegawa M, Kishino H, and Yano T. 1985. Dating of the human-ape splitting by a molecular clock of mitochondrial DNA. J Mol Evol 22:160-174.

Heringa J. 1998. Detection of internal repeats: how common are they? Curr Opin Struct Biol 8:338-345.

Hirano T, Kinoshita N, Morikawa K, and Yanagida M. 1990. Snap helix with knob and hole: essential repeats in S. pombe nuclear protein nuc2+. Cell 60:319-328.

Huelsenbeck JP, and Ronquist F. 2001. MRBAYES: Bayesian inference of phylogenetic trees. Bioinformatics 17:754-755.

Javadi Y, and Itzhaki LS. 2013. Tandem-repeat proteins: regularity plus modularity equals design-ability. Curr Opin Struct Biol 23:622-631.

Jean NL, Bougault CM, Lodge A, Derouaux A, Callens G, Egan AJ, Ayala I, Lewis RJ, Vollmer W, and Simorre JP. 2014. Elongated structure of the outer-membrane activator of peptidoglycan synthesis LpoA: implications for PBP1A stimulation. Structure 22:10471054.

Jernigan KK, and Bordenstein SR. 2014. Ankyrin domains across the Tree of Life. PeerJ 2:e264. JW IJ, Carlson AC, and Kennedy EL. 2007. Anaplasma phagocytophilum AnkA is tyrosinephosphorylated at EPIYA motifs and recruits SHP-1 during early infection. Cell Microbiol 9:1284-1296. 
Kippert F, and Gerloff DL. 2004. Timeless and Armadillo: a link too far (Comment on Vodovar et al. Curr. Biol. (2002) 12, R610-R611). Curr Biol 14:R650-651; author reply R652-653.

Kippert F, and Gerloff DL. 2009. Highly sensitive detection of individual HEAT and ARM repeats with HHpred and COACH. PLoS One 4:e7148.

Kobe B, and Kajava AV. 2000. When protein folding is simplified to protein coiling: the continuum of solenoid protein structures. Trends Biochem Sci 25:509-515.

Krieg NR, Ludwig W, Whitman W, Hedlund BP, Paster BJ, Staley JT, Ward N, Brown D, and Parte A. 2011. Bergey's Manual of Systematic Bacteriology. New York: Springer.

Lee SJ, Sekimoto T, Yamashita E, Nagoshi E, Nakagawa A, Imamoto N, Yoshimura M, Sakai H, Chong KT, Tsukihara T, and Yoneda Y. 2003. The structure of importin-beta bound to SREBP-2: nuclear import of a transcription factor. Science 302:1571-1575.

Liu J, Liu X, Dai L, and Wang G. 2007. Recent progress in elucidating the structure, function and evolution of disease resistance genes in plants. J Genet Genomics 34:765-776.

Maddison WP, and Maddison DR. 2006. Mesquite: A modular system for evolutionary analysis. Version 1.1.

Main ER, Stott K, Jackson SE, and Regan L. 2005. Local and long-range stability in tandemly arrayed tetratricopeptide repeats. Proc Natl Acad Sci U S A 102:5721-5726.

Marcotte EM, Pellegrini M, Yeates TO, and Eisenberg D. 1999. A census of protein repeats. $J$ Mol Biol 293:151-160.

Midford PE, Garland Jr. T, and Maddison WP. 2005. PDAP Package of Mesquite. Version 1.07.

Miles ZD, McCarty RM, Molnar G, and Bandarian V. 2011. Discovery of epoxyqueuosine (oQ) reductase reveals parallels between halorespiration and tRNA modification. Proc Natl Acad Sci U S A 108:7368-7372.

Mosavi LK, Cammett TJ, Desrosiers DC, and Peng ZY. 2004. The ankyrin repeat as molecular architecture for protein recognition. Protein Sci 13:1435-1448.

Parmeggiani F, Pellarin R, Larsen AP, Varadamsetty G, Stumpp MT, Zerbe O, Caflisch A, and Pluckthun A. 2008. Designed armadillo repeat proteins as general peptide-binding scaffolds: consensus design and computational optimization of the hydrophobic core. $J$ Mol Biol 376:1282-1304.

Peifer M, Berg S, and Reynolds AB. 1994. A repeating amino acid motif shared by proteins with diverse cellular roles. Cell 76:789-791.

Ponting CP, Aravind L, Schultz J, Bork P, and Koonin EV. 1999. Eukaryotic signalling domain homologues in archaea and bacteria. Ancient ancestry and horizontal gene transfer. $J \mathrm{Mol}$ Biol 289:729-745.

Reeve J, and Abouheif E. 2003. Phylogenetic Independence. Version 2.0, Department of Biology, McGill University.

Riegler M, Iturbe-Ormaetxe I, Woolfit M, Miller WJ, and O'Neill SL. 2012. Tandem repeat markers as novel diagnostic tools for high resolution fingerprinting of Wolbachia. BMC Microbiol 12 Suppl 1:S12.

Ronquist F, and Huelsenbeck JP. 2003. MrBayes 3: Bayesian phylogenetic inference under mixed models. Bioinformatics 19:1572-1574.

Schaper E, Gascuel O, and Anisimova M. 2014. Deep conservation of human protein tandem repeats within the eukaryotes. Mol Biol Evol 31:1132-1148.

Schlesner M, Miller A, Streif S, Staudinger WF, Müller J, Scheffer B, Siedler F, and Oesterhelt D. 2009. Identification of Archaea-specific chemotaxis proteins which interact with the flagellar apparatus. BMC Microbiol 9:56. 
431 Sedgwick SG, and Smerdon SJ. 1999. The ankyrin repeat: a diversity of interactions on a

432

433

434

435

436

437

438

439

440

441

442

443

444

445

446 common structural framework. Trends Biochem Sci 24:311-316.

Sikorski RS, Boguski MS, Goebl M, and Hieter P. 1990. A repeating amino acid motif in CDC23 defines a family of proteins and a new relationship among genes required for mitosis and RNA synthesis. Cell 60:307-317.

Siozios S, Ioannidis P, Klasson L, Andersson SG, Braig HR, and Bourtzis K. 2013. The diversity and evolution of Wolbachia ankyrin repeat domain genes. PLoS One 8:e55390.

SUPERFAMILY. Available at http://supfam.org/SUPERFAMILY.

Tewari R, Bailes E, Bunting KA, and Coates JC. 2010. Armadillo-repeat protein functions: questions for little creatures. Trends Cell Biol 20:470-481.

Wilson D, Pethica R, Zhou Y, Talbot C, Vogel C, Madera M, Chothia C, and Gough J. 2009. SUPERFAMILY--sophisticated comparative genomics, data mining, visualization and phylogeny. Nucleic Acids Res 37:D380-386.

Zhu B, Nethery KA, Kuriakose JA, Wakeel A, Zhang X, and McBride JW. 2009. Nuclear translocated Ehrlichia chaffeensis ankyrin protein interacts with a specific adenine-rich motif of host promoter and intronic Alu elements. Infect Immun 77:4243-4255. 


\section{1}

Figure 1. ANK, TPR, and ARM repeat structure and incidence across all domains of life

(A) Schematics of the structures of the ANK (Mosavi et al. 2004) , TPR (Main et al. 2005), and ARM (Parmeggiani et al. 2008) repeats. (B) Table indicating the percent of species in each taxonomic domain that contain $\geq 1$ TPR, ARM, or ANK-containing protein.

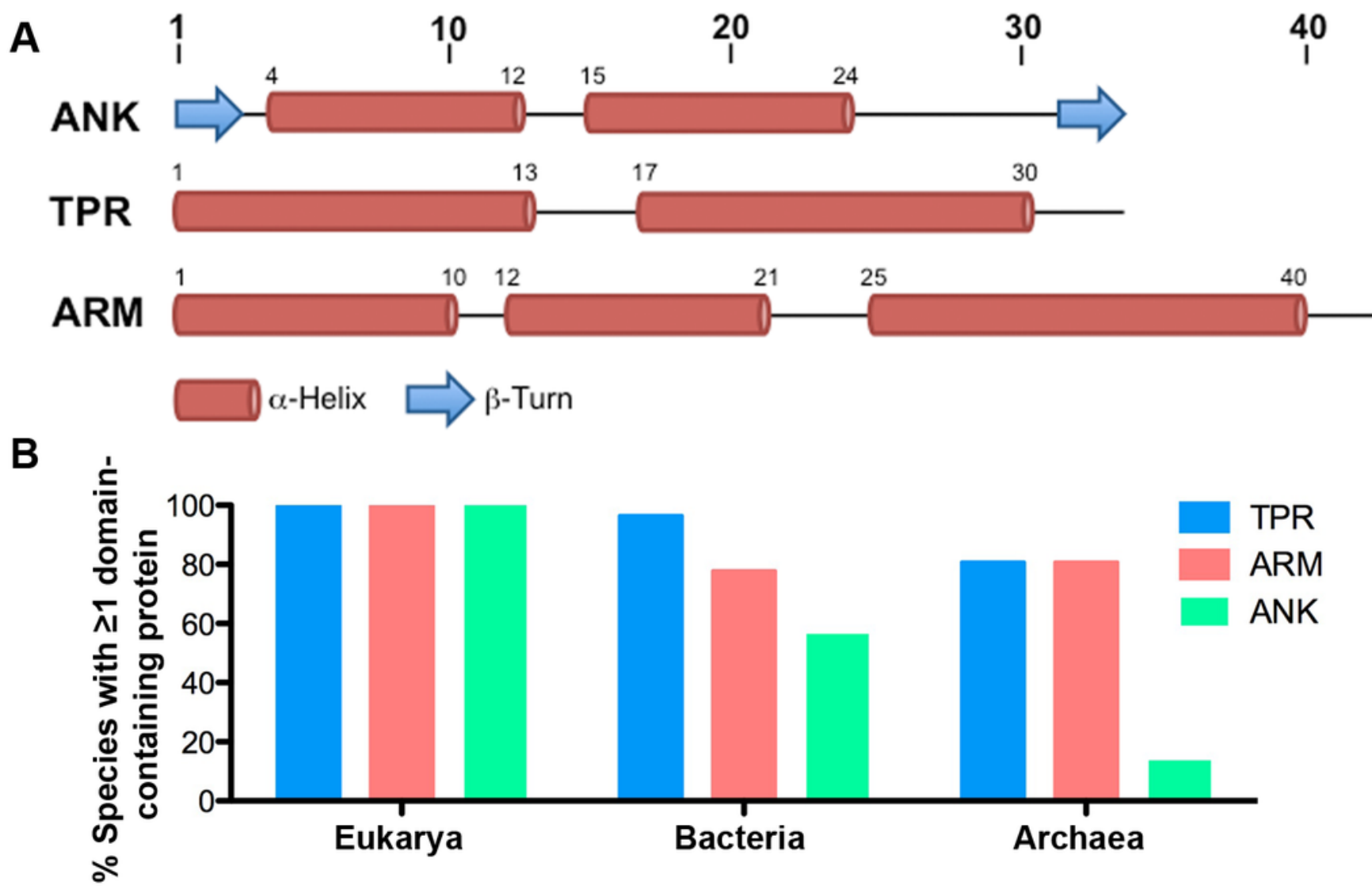




\section{2}

Figure 2. ANK, TPR, and ARM-containing proteins analysis across all domains of life.

Bar graphs of the average percent of the species in the domains. An average percent of ANK, TPR, or ARM-containing proteins for all organisms of the same species was used for these analyses. (A) Eukarya, (B) Bacteria, and (C) Archaea that have one or more ANK, TPR, and ARM-containing proteins. The average percent of protein domain composition is listed below the graph. (A) Mann Whitney U-test $p<0.0001$ for all comparisons, ANOVA $p=5.79 \mathrm{E}-40$. Mann Whitney U-test $p<0.0001$ for all comparisons, ANOVA $p=2.3 \mathrm{E}-203$. (C) Mann Whitney U-test, $p<0.005$ for all comparisons, ANOVA $p=9.85 \mathrm{E}-07$. Error bars represent standard error.
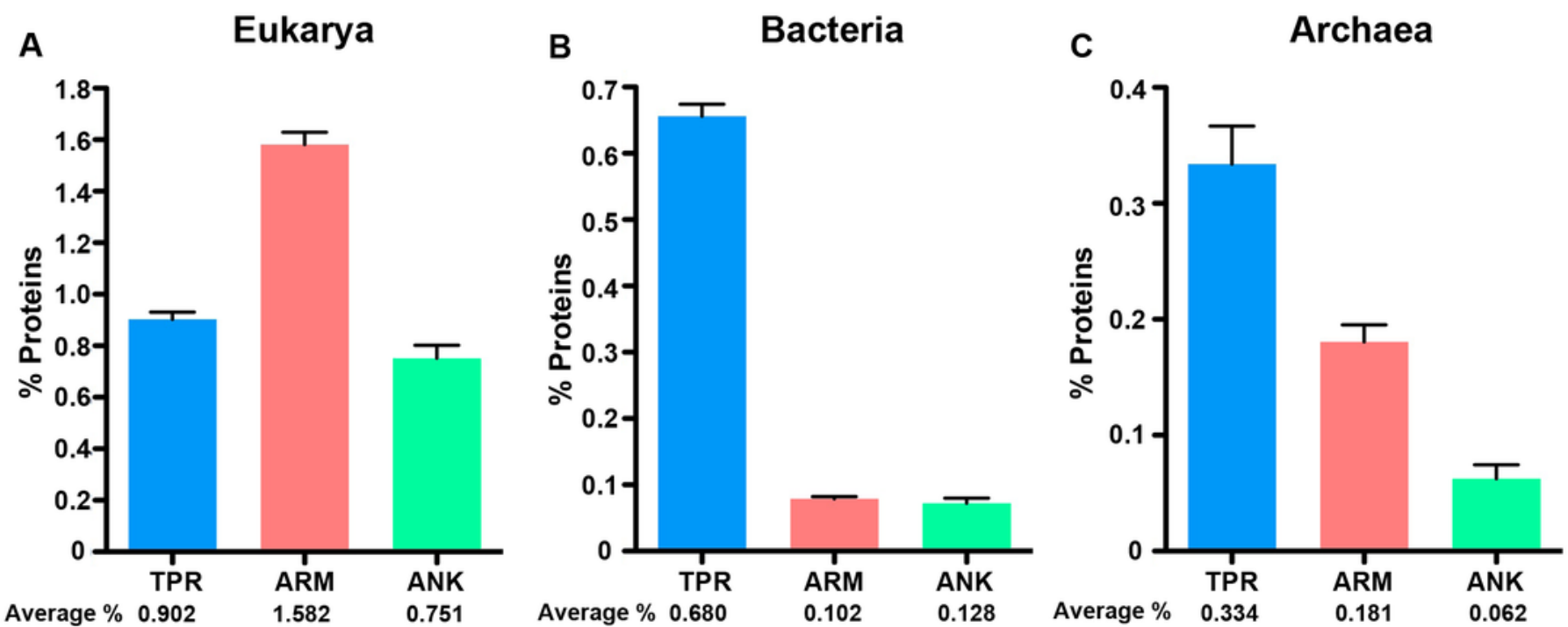


\section{3}

Figure 3. Correlation analysis.

(A) Percent protein composition of TPR, ARM, or ANK-containing proteins in bacterial species and (B) Number of TPR, ARM, or ANK-containing proteins in bacterial species.

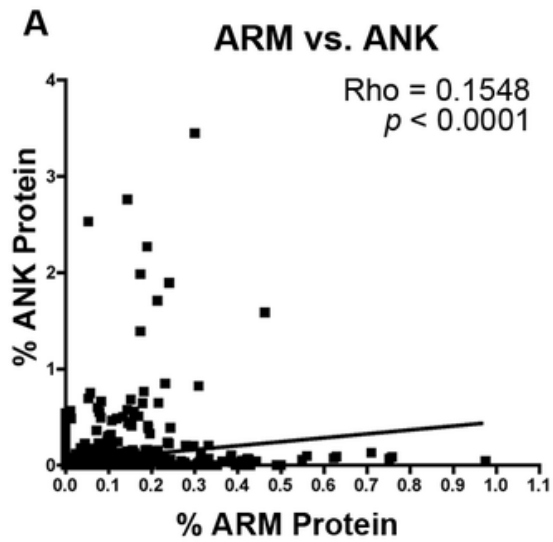

B

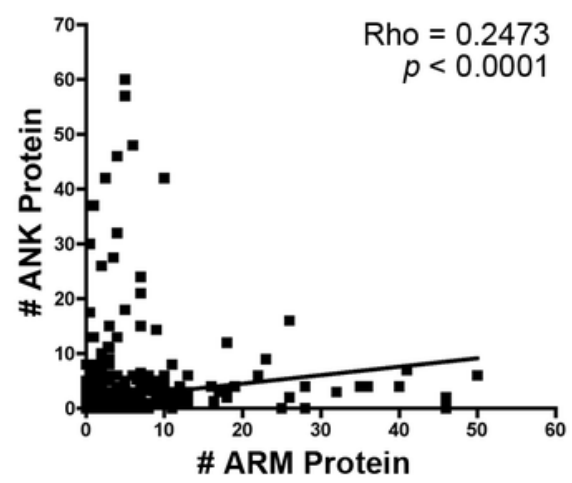

TPR vs. ARM

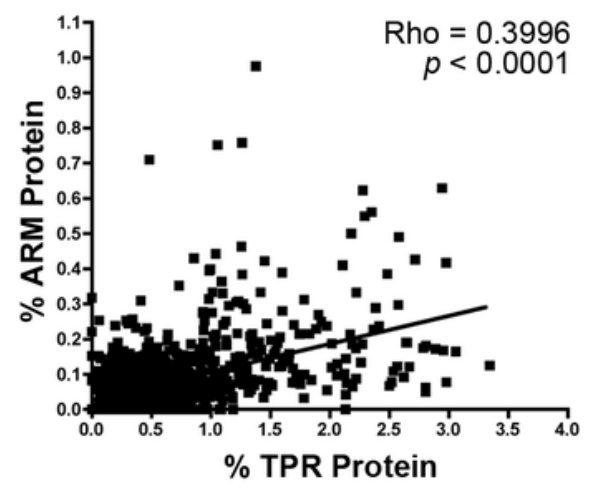

TPR vs. ARM

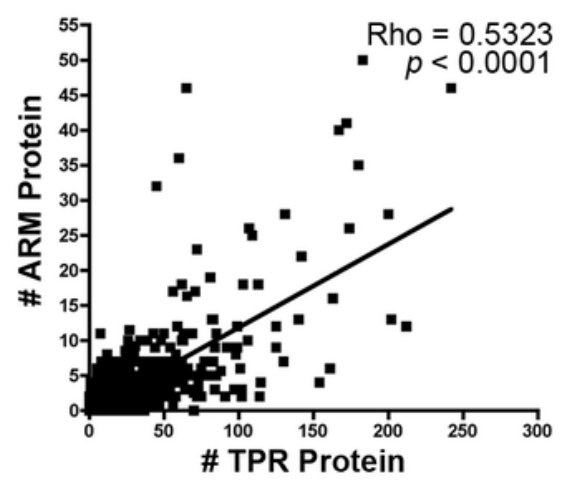

TPR vs. ANK

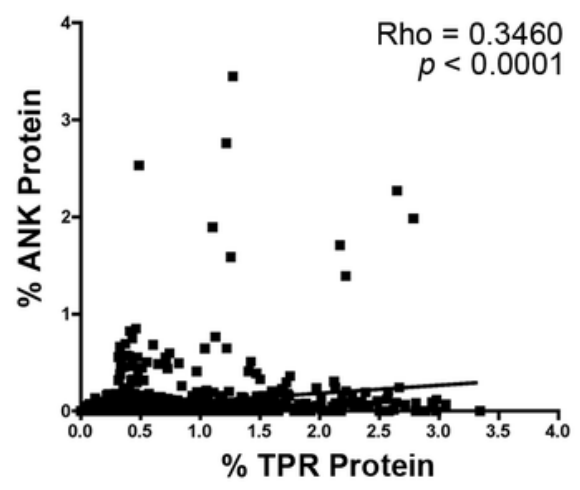

TPR vs. ANK

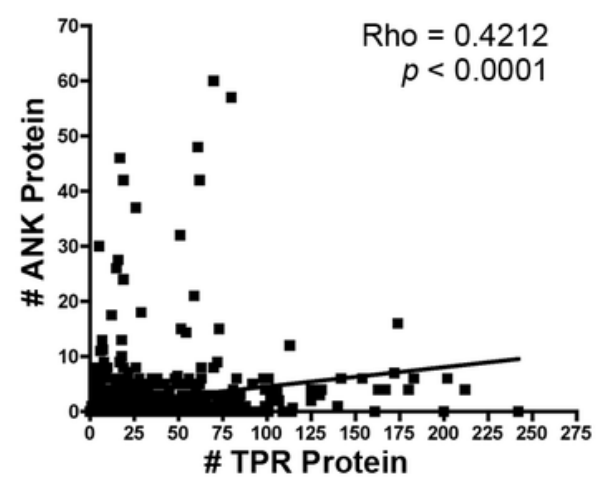


4

Figure 4. TPR, ANK, and ARM-containing proteins analysis across bacterial classes.

Bar graph of the percent of species in bacterial classes that contain $\geq 1$ TPR, ARM, or ANKcontaining proteins (only classes with 5 or more represented species were included in this analysis). Order of classes reflect phylogenetic relationships depicted in Fig. 5A.

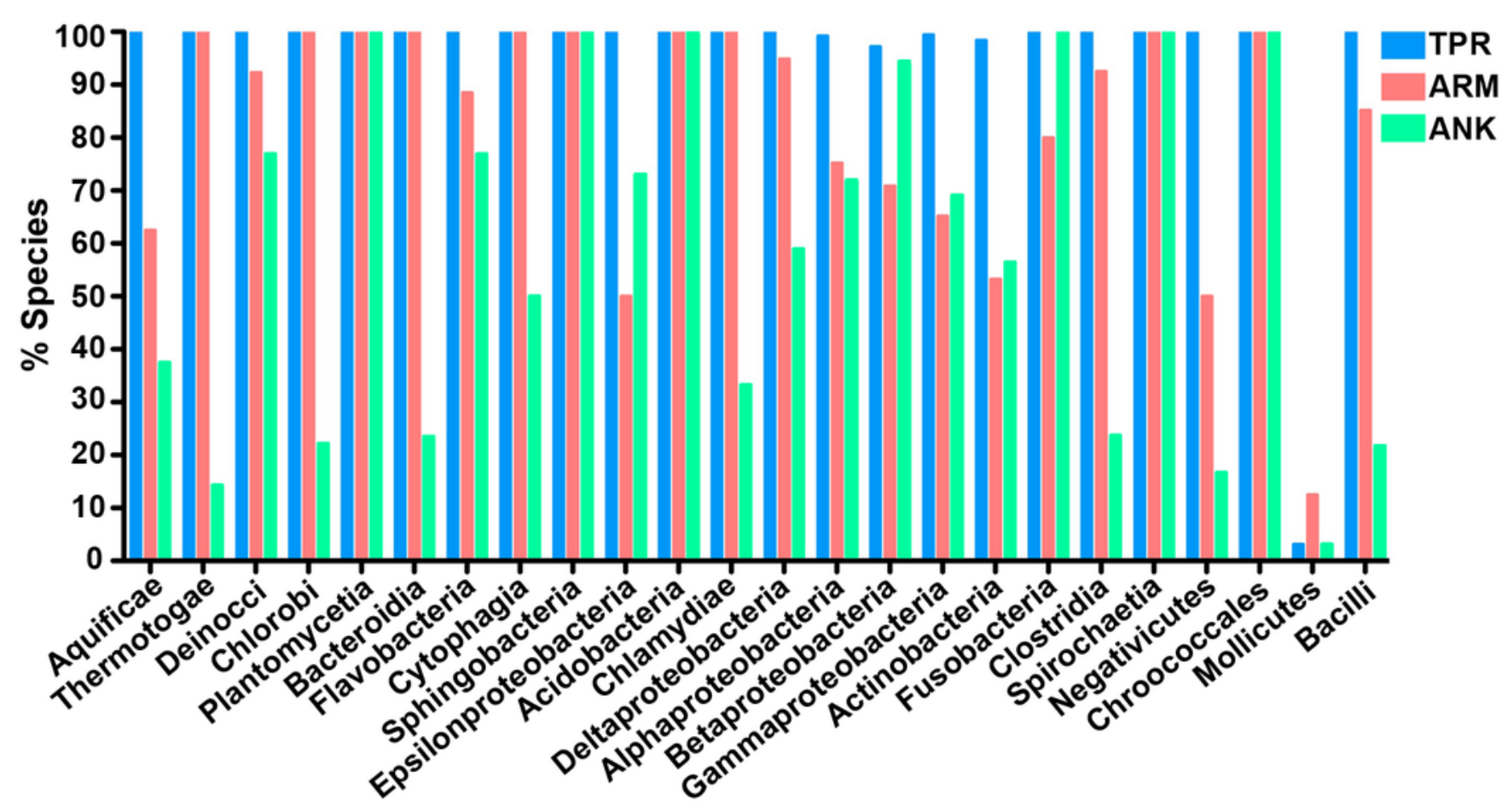




\section{5}

Figure 5. Test for phylogenetic independence of enrichment of ARM, ANK or TPRcontaining proteins in bacteria.

A) Consensus phylogeny of 16S rRNA sequences from one species (randomly selected) in each class. (B) The percent of species per class that fall within the top 15\% most enriched species in the dataset. Only classes with 5 or more represented species were included in this analysis. (C) Spearman correlation analysis of the percent of species within a bacterial classes enriched in each domain. 
A

$16 S$ rRNA Gene Phylogeny

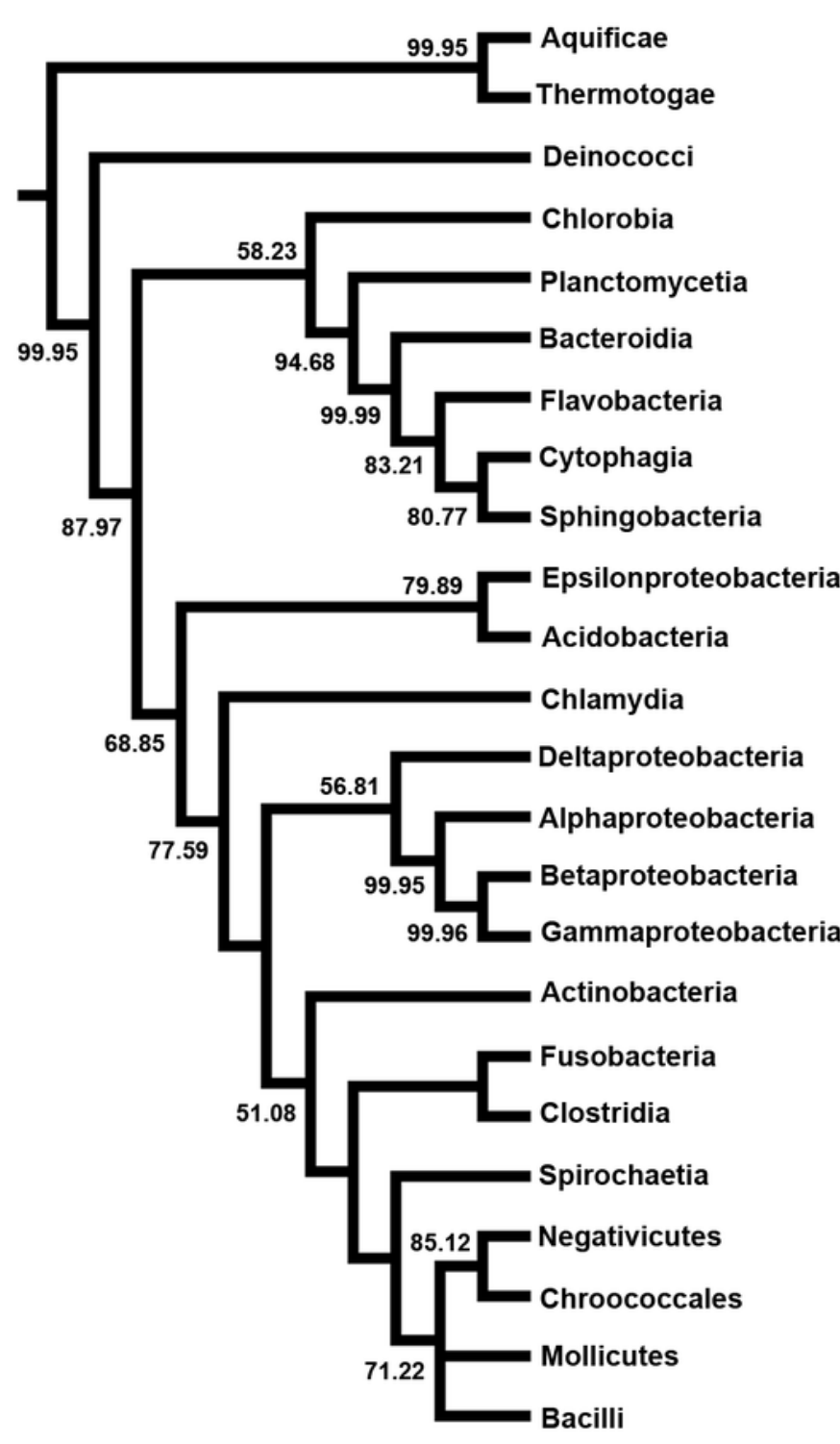

B

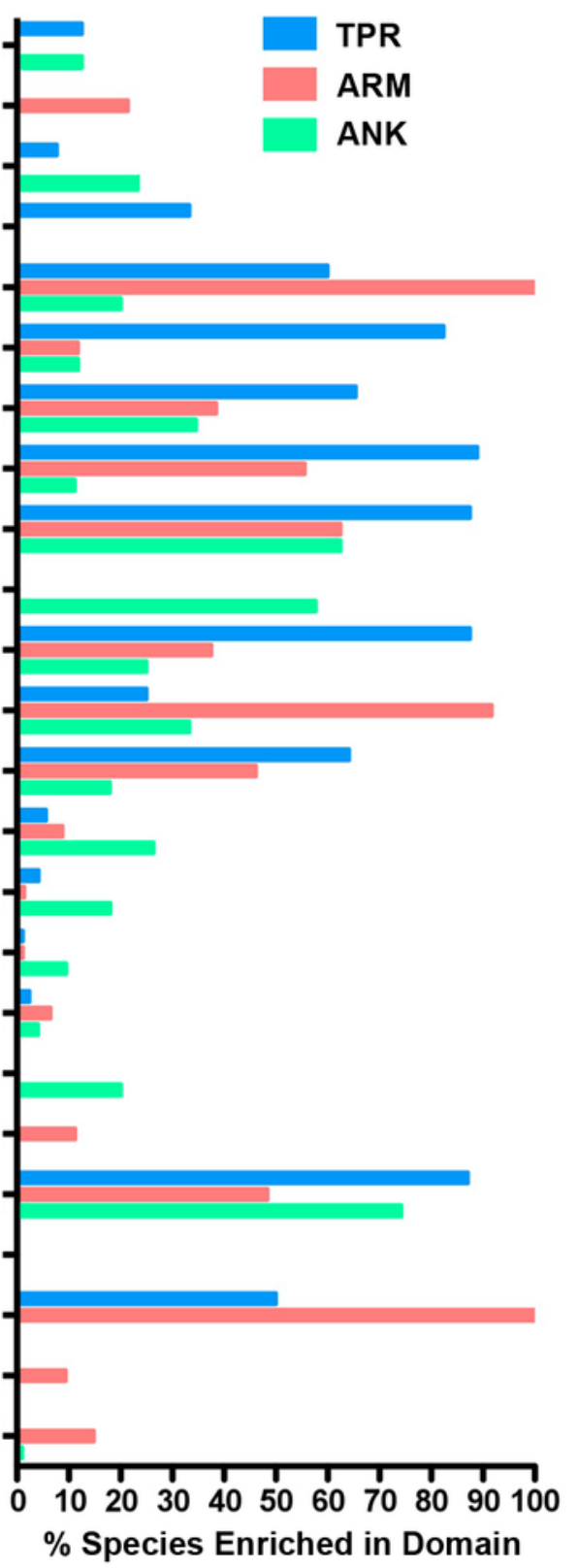

C ARM vS TPR
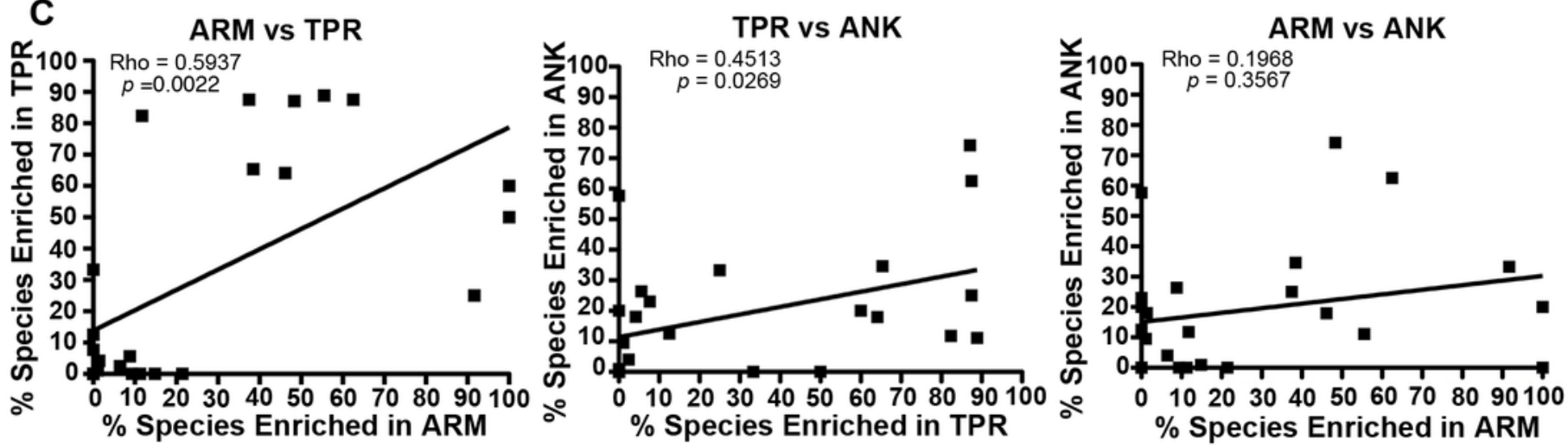


\section{6}

Figure 6. Lifestyle analysis of bacterial species enriched for ARM-containing proteins.

(A-C) Error bars represent standard error and $* \mathrm{P}<0.05, * * \mathrm{P}<0.01$, **P $<0.0001$. (A) Bar graph of the average number of ARM-containing proteins in species of free-living (FL), facultative host-associated (FHA) and obligate intracellular (O) bacteria for those species with at least $0.2 \%$ of their proteome composed of ARM-containing proteins. (2-tailed MannWhitney U; ANOVA, $\mathrm{P}=0.00005$ ). (B) Bar graph of the average percent of the proteome composed of ARM-containing proteins in species of $\mathrm{FL}, \mathrm{FHA}$ and $\mathrm{O}$ bacteria with at least $0.2 \%$ of their proteome composed of ARM-containing proteins. (All comparisons, $\mathrm{P}>0.05$, 2-tailed Mann-Whitney U; ANOVA, $P=0.446$ ). (C) Bar graph of the average number of proteins in the proteomes of species of $\mathrm{FL}, \mathrm{FHA}$ and $\mathrm{O}$ bacteria with at least $0.2 \%$ of their proteome composed of ARM-containing proteins (2-tailed Mann-Whitney U; ANOVA, $\mathrm{P}=1.98 \mathrm{E}-10$ ).
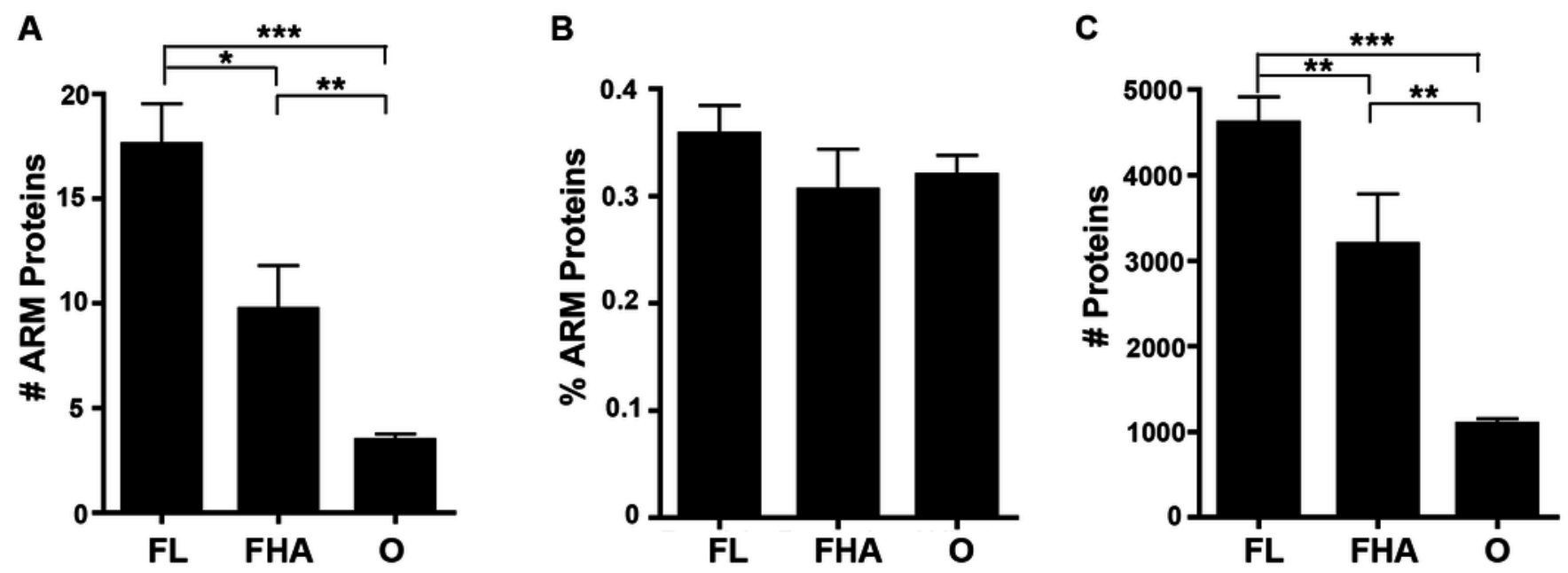